\title{
A Diffusive Limit for the Profiles of Random Young Diagrams by Way of Free Probability
}

\author{
Dedicated to Professor Takeshi Hirai on his 80th birthday
}

by

\author{
Akihito HorA
}

\begin{abstract}
Concentration phenomena in statistical ensembles of Young diagrams have been investigated as static models first for the Plancherel ensemble by Vershik-Kerov and LoganShepp in 1970s and later in some other group-theoretical setting by Biane. On the other hand, a dynamical model of concentration for Young diagrams, which is not directly connected with group representations, was shown by Funaki-Sasada in the framework of hydrodynamic limit. The aim of this paper is to present a new dynamical model of concentration for Young diagrams featuring the group-theoretical sense. Starting from an initial state yielding concentration and a microscopic dynamics keeping the Plancherel measure invariant, we derive an evolution of the profiles of Young diagrams under a diffusive scaling limit. The resulting evolution along macroscopic time is described in terms of the notions of Voiculescu's free probability theory such as free compression and free convolution of Kerov transition measures.
\end{abstract}

2010 Mathematics Subject Classification: Primary 82C41; Secondary 20C30, 46L54, $60 \mathrm{~J} 27$.

Keywords: diffusive limit, Young diagram, symmetric group, free probability, Markov chain, Plancherel measure.

\section{$\S 1$. Introduction}

The set of Young diagrams of size $n$, which is denoted by $\mathbb{Y}_{n}$, parametrizes all the equivalence classes of irreducible representations of the symmetric group $\mathfrak{S}_{n}$. The Plancherel measure $\mathbb{M}_{\mathrm{Pl}}^{(n)}$ is a fundamental probability measure on $\mathbb{Y}_{n}$, which is

Communicated by T. Kumagai. Received May 26, 2014. Revised January 22, 2015, March 10, 2015, and June 5, 2015.

A. Hora: Department of Mathematics, Hokkaido University, Sapporo 060-0810, Japan; e-mail: hora@math.sci.hokudai.ac.jp

(C) 2015 Research Institute for Mathematical Sciences, Kyoto University. All rights reserved. 
defined by

$$
\mathbb{M}_{\mathrm{Pl}}^{(n)}(\lambda)=\frac{(\operatorname{dim} \lambda)^{2}}{n !}, \quad \lambda \in \mathbb{Y}_{n}
$$

where $\operatorname{dim} \lambda$ denotes the dimension of an irreducible representation of $\mathfrak{S}_{n}$ labeled by $\lambda \in \mathbb{Y}_{n}$. We call the probability space $\left(\mathbb{Y}_{n}, \mathbb{M}_{\mathrm{Pl}}^{(n)}\right)$ the Plancherel ensemble of Young diagrams of size $n$, anticipating that it is a certain equilibrium state in our context. It is known that a Young diagram chosen out of the Plancherel ensemble at random, strictly speaking according to the probability designed by $\mathbb{M}_{\mathrm{Pl}}^{(n)}$, should always look like a special shape. This is the famous phenomenon of concentration to the limit shape discovered by Logan-Shepp [9] and Vershik-Kerov [13]. A more precise statement is formulated as a weak law of large numbers as follows. Let us identify a Young diagram $\lambda \in \mathbb{Y}_{n}$ with its profile as defined in Subsection 2.1 (see Figure 1). We consider the function $\lambda$ rescaled by $1 / \sqrt{n}$ :

$$
\lambda^{\sqrt{n}}(x)=\frac{1}{\sqrt{n}} \lambda(\sqrt{n} x), \quad x \in \mathbb{R} .
$$

Set

$$
\Omega(x)= \begin{cases}\frac{2}{\pi}\left(x \arcsin \frac{x}{2}+\sqrt{4-x^{2}}\right), & |x| \leq 2 \\ |x|, & |x|>2\end{cases}
$$

Then, for any $\epsilon>0$, we have

$$
\lim _{n \rightarrow \infty} \mathbb{M}_{\mathrm{Pl}}^{(n)}\left(\left\{\lambda \in \mathbb{Y}_{n}\left|\sup _{x \in \mathbb{R}}\right| \lambda^{\sqrt{n}}(x)-\Omega(x) \mid \geq \epsilon\right\}\right)=0 .
$$

The Plancherel measure can be lifted to the probability $\mathbb{M}_{\mathrm{Pl}}$ on the space $\mathfrak{T}$ consisting of the infinite paths connecting Young diagrams

$$
t=(t(0)=\emptyset \nearrow t(1)=\square \nearrow t(2) \nearrow \cdots \nearrow t(n) \nearrow \cdots), \quad t(n) \in \mathbb{Y}_{n},
$$

so that it is the $n$th marginal distribution as

$$
\mathbb{M}_{\mathrm{Pl}}^{(n)}(\lambda)=\mathbb{M}_{\mathrm{Pl}}(\{t \in \mathfrak{T} \mid t(n)=\lambda\}), \quad \lambda \in \mathbb{Y}_{n}
$$

Then we can show almost sure convergence to the limit shape $\Omega$ as a strong law of large numbers. This kind of concentration is also observed in some other settings than the Plancherel one. In [1] and [2], Biane pointed out the property of "approximate factorization" for states of the group algebra $\mathbb{C}\left[\mathfrak{S}_{n}\right]$ induced by probabilities on $\mathbb{Y}_{n}$ and gave interesting examples of these concentration phenomena. The approximate factorization property is interpreted as ergodicity in a certain weak sense. The limit shape in the Plancherel ensemble simply means the 1-point function of the system. A thorough treatment including higher correlation functions was developed by Borodin-Okounkov-Olshanski [3]. 
Let us note that the concentration to the limit shape of Young diagrams is a static result under the micro-macro correspondence by the rescaling given by (1.1). The purpose of this paper is to capture the evolution of the rescaled profiles of Young diagrams along macroscopic time under the same micro-macro correspondence. In [5], Funaki-Sasada showed remarkable results on hydrodynamic limit for the evolution of Young diagrams. In the model they treated, the microscopic transition rule governing the dynamics on the set of Young diagrams is given by creation and annihilation of a box according to the uniform distribution conditioned on their size. Although their model is simple and natural, our aim here is to consider a dynamical model featuring the direct group-theoretical meaning of Young diagrams (which label the irreducible representations of symmetric groups). The results of Funaki-Sasada [5] are given in the setting of the grand canonical ensemble under which a Markov chain on the totality of Young diagrams of all sizes allows variation of the number of boxes. The main theorem of this paper is stated in the canonical ensemble setting where we consider a Markov chain on $\mathbb{Y}_{n}$ and then take a limit as $n \rightarrow \infty$. When we discuss the Plancherel ensemble in the grand canonical manner, it seems natural to consider the probability on $\mathbb{Y}=\bigsqcup_{n=0}^{\infty} \mathbb{Y}_{n}$ through poissonization of $\mathbb{M}_{\mathrm{Pl}}^{(n)}$ 's. In Section 4 , we formulate this model and state some aspects of the problems when its scaling limit is considered.

It is interesting that certain Markov chains on the set of Young diagrams are related to queuing networks in which Young diagrams are formed because of existence of the priority structure for the services. We do not intend to discuss the relationship with such dynamical models here but mention [11] and references therein.

The framework of the scaling limit in this paper is as follows. Recall that, if the microscopic Plancherel ensemble is zoomed out under scaling limit by $1 / \sqrt{n}$ as in (1.1), one observes $\Omega$ of (1.2) macroscopically. We consider a continuous time Markov chain $\left(X_{s}^{(n)}\right)_{s \geq 0}$ on the state space $\mathbb{Y}_{n}$ which keeps the Plancherel measure $\mathbb{M}_{\mathrm{Pl}}^{(n)}$ invariant. Then, if the chain starts with the initial state $\mathbb{M}_{\mathrm{Pl}}^{(n)}$, the macroscopic shape remains $\Omega$ as time goes by. As an initial state on $\mathbb{Y}_{n}$ let us now take a probability $\mathbb{M}_{0}^{(n)}$ under which we know in advance that concentration occurs as $n \rightarrow \infty$. After $1 / \sqrt{n}$-scaling limit as in (1.1) with respect to $\mathbb{M}_{0}^{(n)}$, a certain macroscopic shape $\omega_{0}$ is hence observed. When we derive the same Markov chain as above from the initial state $\mathbb{M}_{0}^{(n)}$, it is expected that the distribution $\mathbb{M}_{s}^{(n)}$ on $\mathbb{Y}_{n}$ at time $s$ will tend to $\mathbb{M}_{\mathrm{Pl}}^{(n)}$ as $s \rightarrow \infty$. Then, seen from the macroscopic point of view, an evolution from $\omega_{0}$ to $\Omega$ should be observed. Let us make the scale more precise. We assume that the microscopic state $\left(\mathbb{Y}_{n}, \mathbb{M}_{0}^{(n)}\right)$ appears if the macroscopic initial shape $\omega_{0}$ is zoomed in $\sqrt{n}$ multiple. Given a macroscopic 
time $t>0$, we consider the situation after microscopically long time $s=t n$ and, observing it by the rescale of $1 / \sqrt{n}$, see some macroscopic shape $\omega_{t}$ at time $t$. Note that the scale of time versus space is the diffusive one. Our aim is to prove this concentration to $\omega_{t}$ at each time $t$ and to describe $\omega_{t}$ as explicitly as possible as a function of $t$. Since we have convergence to the Plancherel measure as $s \rightarrow \infty$ in the microscopic situation, $\omega_{t}$ should converge to the limit shape $\Omega$ as $t \rightarrow \infty$.

Let us introduce some notions needed to state our main theorem which realizes the above framework. In order to take a Markov chain on $\mathbb{Y}_{n}$ which keeps $\mathbb{M}_{\mathrm{Pl}}^{(n)}$ invariant, we consider (a special case of) the down and up transition probabilities used in [4]. For two Young diagrams $\lambda$ and $\mu$ with sizes satisfying $|\mu|+1=|\lambda|$, we use the notation of $\mu \nearrow \lambda$ if $\lambda$ is formed by adding one box to $\mu$. For $\lambda \in \mathbb{Y}_{n}$ and $\mu \in \mathbb{Y}_{n-1}(n \in \mathbb{N})$, set

$$
P_{\lambda \mu}^{\downarrow}=\left\{\begin{array}{ll}
\frac{\operatorname{dim} \mu}{\operatorname{dim} \lambda}, & \mu \nearrow \lambda, \\
0, & \text { otherwise, }
\end{array} P_{\mu \lambda}^{\uparrow}= \begin{cases}\frac{\operatorname{dim} \lambda}{n \operatorname{dim} \mu}, & \mu \nearrow \lambda, \\
0, & \text { otherwise. }\end{cases}\right.
$$

The branching rules of restriction and induction

$$
\operatorname{Res}_{\mathfrak{S}_{n-1}}^{\mathfrak{S}_{n}} \lambda \cong \bigoplus_{\mu \in \mathbb{Y}_{n-1}: \mu \nearrow \lambda} \mu, \quad \operatorname{Ind}_{\mathfrak{S}_{n-1}}^{\mathfrak{S}_{n}} \mu \cong \bigoplus_{\lambda \in \mathbb{Y}_{n}: \mu \nearrow \lambda} \lambda
$$

imply that $P^{\downarrow}$ and $P^{\uparrow}$ satisfy

$$
\sum_{\mu \in \mathbb{Y}_{n-1}} P_{\lambda \mu}^{\downarrow}=1, \quad \sum_{\lambda \in \mathbb{Y}_{n}} P_{\mu \lambda}^{\uparrow}=1
$$

Hence, setting $P^{(n)}=P^{\downarrow} P^{\uparrow}$, that is,

$$
P_{\lambda \mu}^{(n)}=\sum_{\nu \in \mathbb{Y}_{n-1}} P_{\lambda \nu}^{\downarrow} P_{\nu \mu}^{\uparrow}, \quad \lambda, \mu \in \mathbb{Y}_{n}
$$

we obtain a stochastic matrix $P^{(n)}$ of degree $\left|\mathbb{Y}_{n}\right|$. We have

$$
P_{\lambda \mu}^{(n)}= \begin{cases}\mid\{\text { peaks of } \lambda\} \mid / n, & \lambda=\mu, \\ \operatorname{dim} \mu /(n \operatorname{dim} \lambda), & \lambda \wedge \mu \in \mathbb{Y}_{n-1}, \\ 0, & \text { otherwise, }\end{cases}
$$

for $\lambda, \mu \in \mathbb{Y}_{n}$. Here if $\lambda, \mu \in \mathbb{Y}_{n}$ are distinct, then there can exist at most one Young diagram $\nu \in \mathbb{Y}_{n-1}$ satisfying both $\nu \nearrow \lambda$ and $\nu \nearrow \mu$, namely $\nu=\lambda \wedge \mu$ (= the set-theoretical intersection of the boxes). The number of Young diagrams $\nu \in \mathbb{Y}_{n-1}$ satisfying $\nu \nearrow \lambda$ equals the number of peaks of $\lambda$ (see (2.1)). More generally, let $G$ be a finite group and $H$ its subgroup. We consider the irreducible 
decomposition of the restriction-induction: for $\lambda \in \widehat{G}$,

$$
\operatorname{Ind}_{H}^{G} \operatorname{Res}_{H}^{G} \lambda \cong \operatorname{Ind}_{H}^{G}\left(\bigoplus_{\nu \in \widehat{H}}\left[c_{\lambda, \nu}\right] \nu\right) \cong \bigoplus_{\mu \in \widehat{G}}\left[\sum_{\nu \in \widehat{H}} c_{\lambda, \nu} c_{\mu, \nu}\right] \mu
$$

where $c_{\lambda, \nu}=\left[\operatorname{Res}_{H}^{G} \lambda, \nu\right]=\left[\operatorname{Ind}_{H}^{G} \nu, \lambda\right]$. Taking the dimension of (1.6), we have

$$
P_{\lambda \mu}=\frac{\operatorname{dim} \mu}{[G: H] \operatorname{dim} \lambda} \sum_{\nu \in \widehat{H}} c_{\lambda, \nu} c_{\mu, \nu}, \quad \lambda, \mu \in \widehat{G} .
$$

The transition probability (1.4) or (1.5) is a special case of (1.7) for $G=\mathfrak{S}_{n}$ and $H=\mathfrak{S}_{n-1}$. The following is a direct consequence of (1.7) and (1.8).

Lemma 1.1. The Plancherel measure $\mathbb{M}_{\mathrm{Pl}}^{G}$ on $\widehat{G}$ :

$$
\mathbb{M}_{\mathrm{Pl}}^{G}(\lambda)=(\operatorname{dim} \lambda)^{2} /|G|, \quad \lambda \in \widehat{G},
$$

is symmetric with respect to $P$ of (1.7), namely we have

$$
\mathbb{M}_{\mathrm{Pl}}^{G}(\lambda) P_{\lambda \mu}=\mathbb{M}_{\mathrm{Pl}}^{G}(\mu) P_{\mu \lambda}, \quad \lambda, \mu \in \widehat{G} .
$$

Hence the restriction-induction Markov chain on $\widehat{G}$ with transition probability matrix $P$ keeps $\mathbb{M}_{\mathrm{Pl}}^{G}$ invariant.

Let us consider a continuous time Markov chain $\left(X_{s}^{(n)}\right)_{s \geq 0}$ with the transition matrix $P^{(n)}$ on the state space $\mathbb{Y}_{n}$. The induced probability on the set of functions (= paths) from $[0, \infty)$ to $\mathbb{Y}_{n}$ is denoted by $\mathcal{M}^{(n)}$. Let $\mathbb{M}_{0}^{(n)}$ be the initial distribution on $\mathbb{Y}_{n}$. The distribution $\mathcal{M}^{(n)}\left(X_{s}^{(n)}=\cdot\right)$ at time $s$ is given by

$$
\mathcal{M}^{(n)}\left(X_{s}^{(n)}=\mu\right)=\sum_{\lambda \in \mathbb{Y}_{n}} \mathbb{M}_{0}^{(n)}(\lambda)\left(e^{s\left(P^{(n)}-I\right)}\right)_{\lambda \mu}, \quad \mu \in \mathbb{Y}_{n}
$$

By identifying a Young diagram with its profile, consider the space $\mathbb{D}$ consisting of the continuous diagrams such that $\mathbb{Y} \subset \mathbb{D}$. Here $\omega \in \mathbb{D}$ is by definition a function $\mathbb{R} \rightarrow \mathbb{R}$ satisfying the following conditions:

$$
\begin{aligned}
& |\omega(x)-\omega(y)| \leq|x-y|, \quad x, y \in \mathbb{R}, \\
& \omega(x)=|x| \quad \text { if }|x| \text { is large enough. }
\end{aligned}
$$

Let $\mathfrak{m}_{\omega}$ denote the transition measure of $\omega \in \mathbb{D}$ (see (2.2) and (2.3)). The support $\operatorname{supp} \omega=\operatorname{supp} \mathfrak{m}_{\omega}$ is defined as usual. The transition measure of $\Omega$ in (1.2) is the standard semi-circle distribution such that

$$
\mathfrak{m}_{\Omega}(d x)=\frac{1}{2 \pi} \sqrt{4-x^{2}} 1_{[-2,2]}(x) d x .
$$


For a probability on $\mathbb{R}$, its $k$ th moment and free cumulant are denoted by $M_{k}(\cdot)$ and $R_{k}(\cdot)$ respectively for $k \in \mathbb{N}$ (see Subsection 2.2). A decisive fact is that the free cumulant sequence of $(1.12)$ is

$$
\begin{aligned}
& R_{1}\left(\mathfrak{m}_{\Omega}\right)=0, \quad R_{2}\left(\mathfrak{m}_{\Omega}\right)=1, \\
& R_{3}\left(\mathfrak{m}_{\Omega}\right)=R_{4}\left(\mathfrak{m}_{\Omega}\right)=R_{5}\left(\mathfrak{m}_{\Omega}\right)=\cdots=0 .
\end{aligned}
$$

Some observations on topologies on $\mathbb{D}$ are needed to discuss concentration. See Subsection 2.1 for more details. Setting

$$
\mathbb{D}_{a}=\{\omega \in \mathbb{D} \mid \operatorname{supp} \omega \subset(-a, a)\}
$$

for $a>0$, we have $\mathbb{D}=\bigcup_{a>0} \mathbb{D}_{a}$. If $\mathbb{D}$ is equipped with the uniform topology and $\mathbb{D}_{a}$ with the relative topology, we can consider the inductive limit topology on $\mathbb{D}$, which is stronger than the uniform one. An intermediate topology on $\mathbb{D}$ is the moment topology defined by the family of pseudo-distances

$$
\left|\int_{\mathbb{R}} x^{k-1}\left(\omega_{1}(x)-\omega_{2}(x)\right) d x\right|, \quad \omega_{1}, \omega_{2} \in \mathbb{D}, k \in \mathbb{N},
$$

or

$$
\left|M_{k}\left(\mathfrak{m}_{\omega_{1}}\right)-M_{k}\left(\mathfrak{m}_{\omega_{2}}\right)\right|, \quad \omega_{1}, \omega_{2} \in \mathbb{D}, k \in \mathbb{N}
$$

(see Lemma 2.1). The equivalence of (1.14) and (1.15) follows from (2.3).

Now we present a condition for a sequence of probability spaces to form concentration. Expectation with respect to probability $\mathbb{M}$ is denoted by $E_{\mathbb{M}}[\cdot]$.

Definition 1.2. A sequence $\left\{\left(\mathbb{Y}_{n}, \mathbb{M}^{(n)}\right)\right\}_{n \in \mathbb{N}}$ of probability spaces satisfies concentration at $\psi$ if there exists $\psi \in \mathbb{D}$ such that, for any $p \in \mathbb{N}$ and $k_{1}, \ldots, k_{p} \in$ $\{2,3, \ldots\}$,

$$
\lim _{n \rightarrow \infty} E_{\mathbb{M}^{(n)}}\left[M_{k_{1}}\left(\mathfrak{m}_{\lambda \sqrt{n}}\right) \cdots M_{k_{p}}\left(\mathfrak{m}_{\lambda \sqrt{n}}\right)\right]=M_{k_{1}}\left(\mathfrak{m}_{\psi}\right) \cdots M_{k_{p}}\left(\mathfrak{m}_{\psi}\right)
$$

Since supp $\psi$ is compact, (1.16) determines $\psi$ uniquely. We note the following simple facts.

Remark 1.3. (1) The concentration (1.16) implies the weak law of large numbers with respect to the moment topology on $\mathbb{D}$ : for any $\epsilon>0$ and $k \in \mathbb{N}$,

$$
\lim _{n \rightarrow \infty} \mathbb{M}^{(n)}\left(\left\{\lambda \in \mathbb{Y}_{n}|| \int_{\mathbb{R}} x^{k-1}\left(\lambda^{\sqrt{n}}(x)-\psi(x)\right) d x \mid \geq \epsilon\right\}\right)=0 .
$$

(2) If there exist $a>0$ and $n_{0} \in \mathbb{N}$ such that, if $n>n_{0}$, then $\operatorname{supp} \lambda^{\sqrt{n}} \subset$ $(-a, a)$ for all $\lambda$ in supp $\mathbb{M}^{(n)}$ (the so-called balanced condition), in addition to the weak law of large numbers (1.17), then (1.16) is fulfilled. 
Here is our main theorem. The free convolution of two probabilities $\mu$ and $\nu$ on $\mathbb{R}$ is denoted by $\mu \boxplus \nu$. For a probability $\mu$ on $\mathbb{R}$ and $c \in(0,1]$, let $\mu_{c}$ denote the probability on $\mathbb{R}$ obtained as free compression by a projection of expectation $c$ (see Subsection 2.2).

Theorem 1.4. For the Markov chain $\left(X_{s}^{(n)}\right)_{s \geq 0}$ of (1.9), assume that the initial probability spaces $\left\{\left(\mathbb{Y}_{n}, \mathbb{M}_{0}^{(n)}\right)\right\}_{n \in \mathbb{N}}$ satisfy the concentration $(1.16)$ at $\psi=\omega_{0} \in \mathbb{D}$. For any (macroscopic time) $t \geq 0$, consider the distribution of the Markov chain at $s=t n$ :

$$
\mathbb{M}_{t}^{(n)}(\lambda)=\mathcal{M}^{(n)}\left(X_{t n}^{(n)}=\lambda\right), \quad \lambda \in \mathbb{Y}_{n}
$$

Then $\left\{\left(\mathbb{Y}_{n}, \mathbb{M}_{t}^{(n)}\right)\right\}_{n \in \mathbb{N}}$ satisfy concentration at $\omega_{t} \in \mathbb{D}:$ for any $p \in \mathbb{N}$ and $k_{1}, \ldots, k_{p}$ $\in\{2,3, \ldots\}$,

$$
\lim _{n \rightarrow \infty} E_{\mathbb{M}_{t}^{(n)}}\left[M_{k_{1}}\left(\mathfrak{m}_{\lambda \sqrt{n}}\right) \cdots M_{k_{p}}\left(\mathfrak{m}_{\lambda \sqrt{n}}\right)\right]=M_{k_{1}}\left(\mathfrak{m}_{\omega_{t}}\right) \cdots M_{k_{p}}\left(\mathfrak{m}_{\omega_{t}}\right)
$$

for any $t \geq 0$. Here the macroscopic profile $\omega_{t} \in \mathbb{D}$ at time $t$ is characterized through its transition measure $\mathfrak{m}_{\omega_{t}}$ by the following operations of free compression and free convolution:

$$
\mathfrak{m}_{\omega_{t}}=\left(\mathfrak{m}_{\omega_{0}}\right)_{e^{-t}} \boxplus\left(\mathfrak{m}_{\Omega}\right)_{1-e^{-t},} \quad t>0 .
$$

Remark 1.5. In terms of a free cumulant sequence ${ }^{1},(1.18)$ is translated into

$$
\begin{aligned}
& R_{1}\left(\mathfrak{m}_{\omega_{t}}\right)=0, \quad R_{2}\left(\mathfrak{m}_{\omega_{t}}\right)=1, \\
& R_{k}\left(\mathfrak{m}_{\omega_{t}}\right)=R_{k}\left(\mathfrak{m}_{\omega_{0}}\right) e^{-(k-1) t}, \quad k \geq 3
\end{aligned}
$$

(see (2.6) and (2.4) for the free cumulants obtained by free compression and free convolution). Since for any $k \in \mathbb{N}$ we have

$$
\lim _{t \rightarrow \infty} R_{k}\left(\mathfrak{m}_{\omega_{t}}\right)=R_{k}\left(\mathfrak{m}_{\Omega}\right)
$$

by $(1.19), \omega_{t}$ converges to $\Omega$ in $\mathbb{D}$ as $t \rightarrow \infty$ in the macroscopic point of view.

Remark 1.6. If we adopt $P^{\uparrow} P^{\downarrow}$ instead of $P^{\downarrow} P^{\uparrow}$ as the transition matrix of a Markov chain on $\mathbb{Y}_{n}$ governing the microscopic dynamics, we still have invariance of $\mathbb{M}_{\mathrm{Pl}}^{(n)}$ and the same result for the diffusive limit. See Remark 3.6.

For hydrodynamic limit in most models including [5], a fundamental task is to describe the evolution along macroscopic time by specifying a (non-linear) partial differential equation for $\omega_{t}$ obtained through the scaling limit. The profile $\omega_{t}$ is regarded as the interface of the region between $y=\omega_{t}(x)$ and $y=|x|$ whose area is constant in $t$. In the context of this paper, the shape of an element of $\mathbb{D}$ can be

\footnotetext{
${ }^{1}$ Note that, for $\omega_{0}$ also, we have $R_{1}\left(\mathfrak{m}_{\omega_{0}}\right)=0$ and $R_{2}\left(\mathfrak{m}_{\omega_{0}}\right)=1$.
} 
efficiently analyzed by the free cumulant sequence of the transition measure. The correspondence

$$
\omega \in \mathbb{D} \leftrightarrow \mathfrak{m}_{\omega} \leftrightarrow\left\{R_{k}\left(\mathfrak{m}_{\omega}\right)\right\}_{k \in \mathbb{N}}
$$

will be explained in Subsections 2.1 and 2.2. As for a partial differential equation governing the evolution, we derive the one for the Stieltjes transform of $\mathfrak{m}_{\omega_{t}}$ :

$$
G(t, z)=\int_{\mathbb{R}} \frac{1}{z-x} \mathfrak{m}_{\omega_{t}}(d x), \quad z \in \mathbb{C}^{+},
$$

in Theorem 3.3.

The subsequent sections are organized as follows. Section 2 is devoted to reviewing necessary notions and properties. Introduced are the profile of a Young diagram, its transition measure, several notions in free probability theory and so on. The proof of Theorem 1.4 is given in Section 3. In Section 4, we consider the poissonized Plancherel ensemble in which the microscopic dynamics allows variation of sizes of Young diagrams and discuss a setting of the grand canonical ensemble.

\section{$\S 2$. Preliminaries}

\section{§2.1. Young diagram, continuous diagram and transition measure}

A Young diagram is presented by $\lambda=\left(\lambda_{1} \geq \lambda_{2} \geq \cdots \geq \lambda_{l(\lambda)}\right)$ where $l(\lambda)$ denotes the number of rows in $\lambda$ or by $\lambda=\left(1^{m_{1}(\lambda)} 2^{m_{2}(\lambda)} \ldots j^{m_{j}(\lambda)} \ldots\right)$ where $m_{j}(\lambda)$ denotes the number of rows of length $j$ in $\lambda$. In this paper we mainly display a Young diagram by loading the region $y>|x|$ of the $x y$-plane with squares (= boxes) of edge length $\sqrt{2}$ as in Figure 1. The piecewise linear graph presented by bold lines in Figure 1 is called the profile of the Young diagram. A Young diagram identified

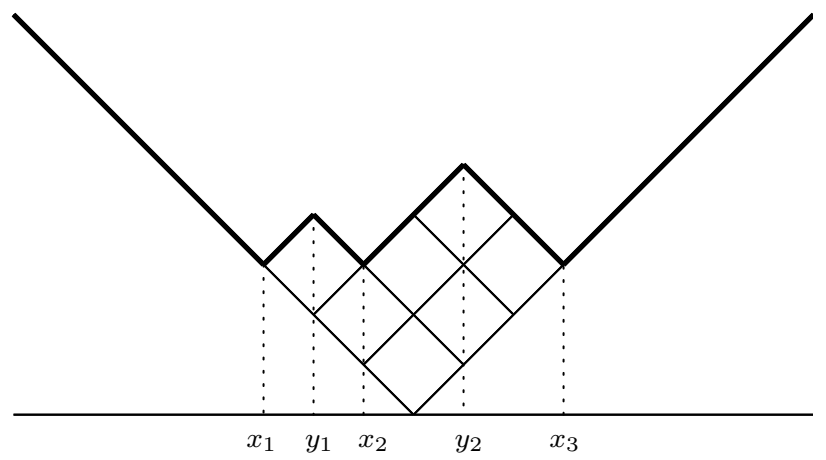

Figure 1. Young diagram, its profile and coordinates $(-3<-2<-1<1<3)$. 
with its profile is regarded as a continuous diagram defined by (1.10) and (1.11). Setting $\mathbb{D}_{0}=\left\{\lambda \in \mathbb{D} \mid \lambda\right.$ is piecewise linear, $\left.\lambda^{\prime}(x)= \pm 1\right\}$, we have $\mathbb{Y} \subset \mathbb{D}_{0} \subset \mathbb{D}$. We encode $\lambda \in \mathbb{D}_{0}$ by using the $x$-coordinates of the valleys and peaks of $\lambda$,

$$
x_{1}<y_{1}<x_{2}<y_{2}<\cdots<x_{r-1}<y_{r-1}<x_{r}
$$

as in Figure 1. Conversely, interlacing $x_{i}$ 's and $y_{i}$ 's as in (2.1) are the coordinates of some $\lambda \in \mathbb{D}_{0}$ if and only if they satisfy

$$
\sum_{i=1}^{r} x_{i}-\sum_{i=1}^{r-1} y_{i}=0
$$

An atomic probability $\mathfrak{m}_{\lambda}$ on $\mathbb{R}$ is uniquely assigned to any $\lambda=\left(x_{1}<y_{1}<\cdots<\right.$ $\left.y_{r-1}<x_{r}\right) \in \mathbb{D}_{0}$ by

$$
\frac{\left(z-y_{1}\right) \cdots\left(z-y_{r-1}\right)}{\left(z-x_{1}\right) \cdots\left(z-x_{r}\right)}=\int_{\mathbb{R}} \frac{1}{z-x} \mathfrak{m}_{\lambda}(d x), \quad z \in \mathbb{C} \backslash \mathbb{R} .
$$

Clearly, supp $\mathfrak{m}_{\lambda}$ coincides with $\left\{x_{1}, \ldots, x_{r}\right\}$. The probability $\mathfrak{m}_{\lambda}$ is called the (Kerov) transition measure of $\lambda$. The transition measure $\mathfrak{m}_{\omega}$ can be defined for any continuous diagram $\omega \in \mathbb{D}$ also through a limiting procedure so that it satisfies

$$
\int_{\mathbb{R}} \frac{1}{z-x} \mathfrak{m}_{\omega}(d x)=\frac{1}{z} \exp \left\{\int_{\mathbb{R}} \frac{1}{x-z}\left(\frac{\omega(x)-|x|}{2}\right)^{\prime} d x\right\}, \quad z \in \mathbb{C} \backslash \mathbb{R},
$$

for $\omega \in \mathbb{D}$. A convenient way is as follows. In fact, (2.3) is satisfied for $\omega \in \mathbb{D}_{0}$. Then, taking a uniformly approximate sequence $\left\{\lambda^{(k)}\right\}_{k \in \mathbb{N}} \subset \mathbb{D}_{0}$ for a given $\omega \in \mathbb{D}$ and recognizing that $\left\{M_{n}\left(\mathfrak{m}_{\lambda^{(k)}}\right)\right\}_{k \in \mathbb{N}}$ forms a Cauchy sequence for any $n \in \mathbb{N}$ by applying (2.3) to $\omega=\lambda^{(k)}$, we use an easy moment problem for a compactly supported probability on $\mathbb{R}$. Conversely, it is known that to a given probability $\mu$ on $\mathbb{R}$ with compact support and mean 0 , there corresponds a unique $\omega \in \mathbb{D}$ such that $\mu=\mathfrak{m}_{\omega}$.

Including consideration of probabilities with non-compact supports and certain moment conditions, a thorough treatment of interplay between transition measures and continuous diagrams is given in [8]. Recall the moment topology on $\mathbb{D}$ defined by (1.14) or (1.15). We record the following facts about topologies on $\mathbb{D}$.

Lemma 2.1. (1) The pointwise and uniform convergence topologies on $\mathbb{D}$ coincide.

(2) Restricted to $\mathbb{D}_{a}$ for $a>0$, the uniform, moment, and inductive limit topologies coincide.

(3) The moment topology is intermediate between the uniform and inductive limit topologies on $\mathbb{D}$. 
Proof. (1) directly follows from conditions (1.10) and (1.11) defining $\mathbb{D} ;(2)$ and (3) are shown by using (2.3).

\section{$\S 2.2$. Free probability theory}

Let us recall several notions of free probability theory. We refer to [10] for details. Here we mainly pick up combinatorial aspects only for one-dimensional distributions. Consider a $C^{*}$-probability space $(\mathcal{A}, \phi)$ consisting of a unital $C^{*}$-algebra $\mathcal{A}$ and a state $\phi$ of $\mathcal{A}$. Let $\mu, \nu$ be the distributions of self-adjoint $a, b \in \mathcal{A}$ respectively. If $a$ and $b$ are free, the distribution of $a+b$ is denoted by $\mu \boxplus \nu$ and called the (additive) free convolution of $\mu$ and $\nu$. Given compactly supported probabilities $\mu, \nu$ on $\mathbb{R}$, the probability $\mu \boxplus \nu$ is uniquely determined and compactly supported. In terms of free cumulants of probabilities on $\mathbb{R}$, the free convolution is characterized by

$$
R_{k}(\mu \boxplus \nu)=R_{k}(\mu)+R_{k}(\nu), \quad k \in \mathbb{N} .
$$

Here $R_{k}(\mu)$ denotes the $k$ th free cumulant of $\mu$, which is defined (from a combinatorial viewpoint) by the cumulant-moment formula (2.5) below. Let $\mathrm{NC}(n)$ denote the set of non-crossing partitions of $\{1, \ldots, n\}$. The subscripts of cumulants (and moments also) are extended to any partition $\pi=\left(v_{1}, \ldots, v_{l}\right) \in \mathrm{NC}(n)$ by setting

$$
R_{\pi}(\mu)=R_{\left|v_{1}\right|}(\mu) \cdots R_{\left|v_{l}\right|}(\mu)
$$

multiplicatively (where $\left|v_{i}\right|$ denotes the cardinality of block $v_{i}$ ). Then we have

$$
M_{n}(\mu)=\sum_{\pi \in \mathrm{NC}(n)} R_{\pi}(\mu), \quad n \in \mathbb{N} .
$$

The Möbius function of the poset $\mathrm{NC}(n)$ enables us to write an inversion formula of (2.5), which expresses $R_{n}(\mu)$ explicitly in terms of $M_{k}(\mu)$ 's.

If $q \in \mathcal{A}$ is a projection $\left(q^{2}=q=q^{*}\right)$ such that the expectation $\phi(q) \neq 0$, setting $\mathcal{B}=q \mathcal{A} q$ and $c=\phi(q)$, we have a $C^{*}$-probability space $\left(\mathcal{B},\left.\frac{1}{c} \phi\right|_{\mathcal{B}}\right)$. Moreover, if self-adjoint $a \in \mathcal{A}$ and $q$ are free, then the distribution of $q a q$ with respect to $\left.\frac{1}{c} \phi\right|_{\mathcal{B}}$ is called the free compression of the distribution $\mu$ of $a$ and denoted by $\mu_{c}$. Given a compactly supported probability $\mu$ on $\mathbb{R}$ and $c \in(0,1]$, the free compression $\mu_{c}$ of $\mu$ is uniquely determined. In terms of free cumulants, the free compression is characterized by

$$
R_{k}\left(\mu_{c}\right)=c^{k-1} R_{k}(\mu)\left(=\frac{1}{c} R_{k}\left(\mu\left(\frac{1}{c} \cdot\right)\right)\right), \quad k \in \mathbb{N} .
$$


For a compactly supported probability $\mu$ on $\mathbb{R}$, set

$$
K_{\mu}(\zeta)=\frac{1}{\zeta}+\sum_{k=0}^{\infty} R_{k+1}(\mu) \zeta^{k}, \quad \zeta \in \mathbb{C} .
$$

The Taylor series part of (2.7) is Voiculescu's $R$-transform of $\mu$. The Stieltjes transform

$$
G_{\mu}(z)=\int_{\mathbb{R}} \frac{1}{z-x} \mu(d x), \quad z \in \mathbb{C},
$$

of $\mu$ and $K_{\mu}$ of (2.7) are inverse functions of each other in appropriate domains such that $|z| \gg 1 \leftrightarrow|\zeta| \ll 1$

\section{$\S 3$. Concentration and diffusive limit}

\section{§3.1. Proof of Theorem 1.4}

We begin with a simple observation.

Lemma 3.1. Let $G$ be a finite group and $H$ its subgroup. Consider the restrictioninduction chain on $\widehat{G}$ with transition probability $P=\left(P_{\lambda \mu}\right)$ given by $(1.7)$. For any conjugacy class $C$ of $G$, taking the column vector $\left(\tilde{\chi}_{C}^{\lambda}\right)_{\lambda \in \widehat{G}}$, we have

$$
P\left(\tilde{\chi}_{C}\right)=\left(\frac{|C \cap H|}{|C|} \tilde{\chi}_{C}\right) .
$$

Proof. The $\mu$-entry of (3.1) for $\mu \in \widehat{G}$ is computed as

$$
\begin{aligned}
P\left(\tilde{\chi}_{C}\right)(\mu) & =\sum_{\nu \in \widehat{G}} P_{\mu \nu} \tilde{\chi}_{C}^{\nu}=\frac{1}{[G: H] \operatorname{dim} \mu} \sum_{\nu \in \widehat{G}} \sum_{\xi \in \widehat{H}} c_{\mu, \xi} c_{\nu, \xi} \chi_{C}^{\nu} \\
& =\frac{1}{[G: H] \operatorname{dim} \mu} \sum_{\xi \in \widehat{H}} c_{\mu, \xi} \chi_{C}^{\operatorname{Ind}_{H}^{G} \xi}=\frac{1}{[G: H] \operatorname{dim} \mu} \chi_{C}^{\operatorname{Ind}_{H}^{G} \operatorname{Res}_{H}^{G} \mu} .
\end{aligned}
$$

To use the induced character formula, divide $C \cap H$ into conjugacy classes of $H$ as $C \cap H=\bigsqcup_{i} C_{i}$. Then we have

$$
\chi_{C}^{\operatorname{Ind}_{H}^{G} \operatorname{Res}_{H}^{G} \mu}=\sum_{i}[G: H] \frac{\left|C_{i}\right|}{|C|} \chi_{C_{i}}^{\operatorname{Res}_{H}^{G} \mu}=[G: H] \frac{|C \cap H|}{|C|} \chi_{C}^{\mu} .
$$

Combining this with (3.2), we obtain (3.1).

In particular, let $C$ be the conjugacy class of $\mathfrak{S}_{n}$ labeled by $\left(\rho, 1^{n-|\rho|}\right)$ for $\rho \in \mathbb{Y}$. Here $\left(\rho, 1^{n-|\rho|}\right) \in \mathbb{Y}_{n}$ is the Young diagram having $n-|\rho|$ one-box rows besides $\rho$. Then

$$
\frac{\left|C \cap \mathfrak{S}_{n-1}\right|}{|C|}=\frac{(n-1) !\left(n-|\rho|+m_{1}(\rho)\right) !}{n !\left(n-1-|\rho|+m_{1}(\rho)\right) !}=1-\frac{|\rho|-m_{1}(\rho)}{n} .
$$


Hence (3.1) gives

$$
P^{(n)}\left(\tilde{\chi}_{\left(\rho, 1^{n-|\rho|}\right)}\right)=\left(1-\frac{|\rho|-m_{1}(\rho)}{n}\right)\left(\tilde{\chi}_{\left(\rho, 1^{n-|\rho|}\right)}\right),
$$

and hence also

$$
e^{t n\left(P^{(n)}-I\right)}\left(\tilde{\chi}_{\left(\rho, 1^{n-|\rho|}\right)}\right)=e^{-t\left(|\rho|-m_{1}(\rho)\right)}\left(\tilde{\chi}_{\left(\rho, 1^{n-|\rho|}\right)}\right) .
$$

Let us show that $\left\{\left(\mathbb{Y}_{n}, \mathbb{M}_{t}^{(n)}\right)\right\}_{n \in \mathbb{N}}$ at $t>0$ satisfies concentration from Definition 1.2. The connection between the situation at the initial time and the one at a microscopic time $s=t n$ is given by (3.4). However, since (3.4) is expressed in terms of irreducible characters, we need a bridge between the irreducible characters and the moments or (free) cumulants of transition measures. These relations were investigated by Biane [2], who noticed the approximate factorization property for states of the group algebra. A more explicit and systematic development was given by Śniady [12]. We are also much influenced by Ivanov-Olshanski [7]. Since we do not claim novelty in the static aspect in this paper, we omit a detailed procedure here, referring to [12, Definition and Theorem 1].

Taking the assumption of Theorem 1.4 and (3.4) into account, and applying the procedure cited above, we can obtain

$$
\lim _{n \rightarrow \infty} E_{\mathbb{M}_{t}^{(n)}}\left[R_{k_{1}}\left(\mathfrak{m}_{\lambda \sqrt{n}}\right) \cdots R_{k_{p}}\left(\mathfrak{m}_{\lambda \sqrt{n}}\right)\right]=\left(r_{k_{1}} e^{-t\left(k_{1}-1\right)}\right) \cdots\left(r_{k_{q}} e^{-t\left(k_{q}-1\right)}\right)
$$

for any $k_{1}, \ldots, k_{q} \in\{3,4, \ldots\}$ and $k_{q+1}=\cdots=k_{p}=2$.

On the other hand, the formula (2.6) for free compression with (1.13) yields

$$
\begin{aligned}
R_{1}\left(\left(\mathfrak{m}_{\omega_{0}}\right)_{e^{-t}}\right) & =0, & & R_{1}\left(\left(\mathfrak{m}_{\Omega}\right)_{1-e^{-t}}\right)=0, \\
R_{2}\left(\left(\mathfrak{m}_{\omega_{0}}\right)_{e^{-t}}\right) & =e^{-t}, & & R_{2}\left(\left(\mathfrak{m}_{\Omega}\right)_{1-e^{-t}}\right)=1-e^{-t}, \\
R_{k}\left(\left(\mathfrak{m}_{\omega_{0}}\right)_{e^{-t}}\right) & =R_{k}\left(\mathfrak{m}_{\omega_{0}}\right) e^{-(k-1) t}, & & R_{k}\left(\left(\mathfrak{m}_{\Omega}\right)_{1-e^{-t}}\right)=0 \quad(k \geq 3) .
\end{aligned}
$$

Since free convolution is characterized by (2.4), we see that the free cumulant sequence $\left\{R_{k}\left(\left(\mathfrak{m}_{\omega_{0}}\right)_{e^{-t}} \boxplus\left(\mathfrak{m}_{\Omega}\right)_{1-e^{-t}}\right)\right\}_{k \in \mathbb{N}}$ is

$$
0,1, r_{3} e^{-2 t}, \ldots, r_{k} e^{-(k-1) t}, \ldots
$$

This implies that the right hand side of (3.5) is equal to

$$
\prod_{i=1}^{p} R_{k_{i}}\left(\left(\mathfrak{m}_{\omega_{0}}\right)_{e^{-t}} \boxplus\left(\mathfrak{m}_{\Omega}\right)_{1-e^{-t}}\right) .
$$

This completes the proof of Theorem 1.4.

Remark 3.2. We can now also see that if the initial state $\left\{\left(\mathbb{Y}_{n}, \mathbb{M}^{(n)}\right)\right\}$ satisfies the approximate factorization property, then so does $\left\{\left(\mathbb{Y}_{n}, \mathbb{M}_{t}^{(n)}\right)\right\}$ for any $t>0$. 


\section{§3.2. A PDE viewpoint}

Let $\omega_{t}$ be the macroscopic profile at $t$ described in Theorem 1.4. To see another aspect of the time evolution of $\omega_{t}$, we consider the Stieltjes transform $G(t, z)$ of $\mathfrak{m}_{\omega_{t}}$ given in (1.20).

Theorem 3.3. The function $G(t, z)$ satisfies the partial differential equation:

$$
\frac{\partial G(t, z)}{\partial t}=\frac{1}{G(t, z)} \frac{\partial G(t, z)}{\partial z}+G(t, z)-\frac{1}{2} \frac{\partial}{\partial z} G(t, z)^{2} .
$$

Proof. We just verify (3.6) from (1.18). From the expressions (2.7) for $\mu=\mathfrak{m}_{\omega_{0}}$ and $\mathfrak{m}_{\omega_{t}}$ respectively, we have

$$
\begin{aligned}
K_{0}(\zeta) & =K_{\mathfrak{m}_{\omega_{0}}}(\zeta)=\frac{1}{\zeta}+\zeta+\sum_{k=2}^{\infty} R_{k+1}\left(\mathfrak{m}_{\omega_{0}}\right) \zeta^{k} \\
K(t, \zeta) & =K_{\mathfrak{m}_{\omega_{t}}}(\zeta)=\frac{1}{\zeta}+\zeta+\sum_{k=2}^{\infty} R_{k+1}\left(\mathfrak{m}_{\omega_{0}}\right) e^{-k t} \zeta^{k}
\end{aligned}
$$

These yield

$$
K_{0}\left(\zeta e^{-t}\right)=\frac{e^{t}}{\zeta}+\frac{\zeta}{e^{t}}-\frac{1}{\zeta}-\zeta+K(t, \zeta)
$$

Differentiating (3.7) in $t$ and $\zeta$ respectively and eliminating $K_{0}^{\prime}$-terms, we obtain

$$
\frac{\partial K(t, \zeta)}{\partial t}+\zeta \frac{\partial K(t, \zeta)}{\partial \zeta}+\frac{1}{\zeta}-\zeta=0
$$

Since

$$
K(t, G(t, z))=z,
$$

differentiating (3.9) by $t$ and $\zeta$ respectively and then putting them into (3.8), we get the desired equation (3.6).

Using (2.3), we readily reformulate (3.6) in terms of the profile of $\omega_{t}$. Set

$$
\rho(t, x)=\frac{\partial}{\partial x}\left(\frac{\omega_{t}(x)-|x|}{2}\right) .
$$

Let $G[f]=G[f](z)$ denote the Stieltjes transform of an $\mathbb{R}$-valued function $f$.

Corollary 3.4. The function $\rho(t, x)$ satisfies

$$
G\left[\frac{\partial \rho}{\partial t}\right]+1=\left(z e^{G[\rho]}-\frac{1}{z e^{G[\rho]}}\right)\left(G\left[\frac{\partial \rho}{\partial x}\right]+\frac{1}{z}\right) .
$$

Since it is not postulated in advance that $\frac{\partial}{\partial x}\left(\omega_{t}(x)-|x|\right)$ is of bounded variation, the derivative in (3.10) should be given an appropriate interpretation. 
Remark 3.5. Since $\left(\mathfrak{m}_{\Omega}\right)_{t}$ is the semi-circle distribution with mean 0 and variance $t$ on $\mathbb{R}$, its Stieltjes transform $g(t, z)$ satisfies the complex Burgers equation

$$
\frac{\partial g(t, z)}{\partial t}=-\frac{1}{2} \frac{\partial}{\partial z} g(t, z)^{2}
$$

as is well-known and easily derived. For very small $t>0$, the right hand side of (3.6) may be considered as a simple sum of contributions of the initial part $\left(\mathfrak{m}_{\omega_{0}}\right)_{e^{-t}}$ and the stationary part $\left(\mathfrak{m}_{\Omega}\right)_{1-e^{-t}}$ under the approximation $1-e^{-t} \doteqdot t$.

Remark 3.6. If we take $P^{(n)}=P^{\uparrow} P^{\downarrow}$ instead of $P^{\downarrow} P^{\uparrow}$ as a microscopic dynamics, the symmetry with respect to $\mathbb{M}_{\mathrm{Pl}}^{(n)}$ holds and Theorem 1.4 remains valid without any modification. In fact, we have only to recognize that (1.5), (3.3) and (3.4) would be the following:

$$
P_{\lambda \mu}^{(n)}= \begin{cases}\frac{\mid\{\text { valleys of } \lambda\} \mid}{n+1}, & \lambda=\mu, \\ \frac{\operatorname{dim} \mu}{(n+1) \operatorname{dim} \lambda}, & \lambda \vee \mu \in \mathbb{Y}_{n+1}, \\ 0, & \text { otherwise }\end{cases}
$$

for $\lambda, \mu \in \mathbb{Y}_{n}$, where $\lambda \vee \mu$ means the set-theoretic union of the boxes,

$$
\begin{aligned}
& P^{(n)}\left(\tilde{\chi}_{\left(\rho, 1^{n-|\rho|}\right)}\right)=\left(1-\frac{|\rho|-m_{1}(\rho)}{n+1}\right)\left(\tilde{\chi}_{\left(\rho, 1^{n-|\rho|}\right)}\right), \\
& e^{t n\left(P^{(n)}-I\right)}\left(\tilde{\chi}_{\left(\rho, 1^{n-|\rho|}\right)}\right)=e^{-\frac{t n\left(|\rho|-m_{1}(\rho)\right)}{n+1}}\left(\tilde{\chi}_{\left(\rho, 1^{n-|\rho|}\right)}\right)
\end{aligned}
$$

for $\rho \in \mathbb{Y}$ and $n \in \mathbb{N}$.

\section{§4. Grand canonical ensemble}

Given a sequence $\left\{\left(\mathbb{Y}_{n}, \mathbb{M}^{(n)}\right)\right\}_{n \in \mathbb{N}}$ of probability spaces, we set

$$
\mathbb{M}^{(\xi)}=\sum_{n=0}^{\infty} p_{\xi}(n) \mathbb{M}^{(n)}
$$

where $\xi>0$ is a parameter of the system and $p_{\xi}$ is a probability on $\mathbb{N} \sqcup\{0\}$. The unique probability on $\mathbb{Y}_{0}=\{\emptyset\}$ is denoted by $\mathbb{M}^{(0)}$. Then $\mathbb{M}^{(\xi)}$ is a probability on $\mathbb{Y}=\bigsqcup_{n=0}^{\infty} \mathbb{Y}_{n}$. This gives us the formalism of grand canonical ensembles. In what follows, we consider poissonization of the ensemble by taking a Poisson distribution as $p_{\xi}$ :

$$
p_{\xi}=\sum_{n=0}^{\infty} \frac{e^{-\xi} \xi^{n}}{n !} \delta_{n} .
$$


Since $p_{\xi}$ in (4.2) has mean $\xi$ and standard deviation $\sqrt{\xi}$, it tends to concentrate near $n=\xi$ as $\xi \rightarrow \infty$. The concentration (1.16) is inherited by poissonization.

Proposition 4.1. If $\left\{\left(\mathbb{Y}_{n}, \mathbb{M}^{(n)}\right)\right\}_{n \in \mathbb{N}}$ satisfies (1.16), then

$$
\lim _{\xi \rightarrow \infty} E_{\mathbb{M}^{(\xi)}}\left[M_{k_{1}}\left(\mathfrak{m}_{\lambda \sqrt{\xi}}\right) \cdots M_{k_{p}}\left(\mathfrak{m}_{\lambda \sqrt{\xi}}\right)\right]=M_{k_{1}}\left(\mathfrak{m}_{\psi}\right) \cdots M_{k_{p}}\left(\mathfrak{m}_{\psi}\right)
$$

for the poissonization $\mathbb{M}^{(\xi)}$.

Proof. The result follows from an elementary estimate. Expressing (4.3) as a poissonized sum, we divide it, for example, into $\left\{|n-\xi| \leq \xi^{3 / 4}\right\}$ and $\{|n-\xi|$ $\left.>\xi^{3 / 4}\right\}$.

Taking the Plancherel measure $\mathbb{M}_{\mathrm{Pl}}^{(n)}$ as $\mathbb{M}^{(n)}$ and a Poisson distribution $p_{\xi}$ in (4.1), we set

$$
\mathbb{M}_{\mathrm{PP}}^{(\xi)}(\lambda)=\frac{e^{-\xi} \xi^{|\lambda|}(\operatorname{dim} \lambda)^{2}}{(|\lambda| !)^{2}}, \quad \lambda \in \mathbb{Y}
$$

The probability space $\left(\mathbb{Y}, \mathbb{M}_{\mathrm{PP}}^{(\xi)}\right)$ is called the poissonized Plancherel ensemble. Since the Plancherel ensemble $\mathbb{M}_{\mathrm{Pl}}^{(n)}$ satisfies the concentration (1.16) with the macroscopic shape $\Omega$ of (1.2), Proposition 4.1 implies that the limit shape $\Omega$ is observed macroscopically in the poissonized Plancherel ensemble also. In particular,

$$
\lim _{\xi \rightarrow \infty} \mathbb{M}_{\mathrm{PP}}^{(\xi)}\left(\left\{\lambda \in \mathbb{Y}\left|\sup _{x \in \mathbb{R}}\right| \lambda^{\sqrt{\xi}}(x)-\Omega(x) \mid \geq \epsilon\right\}\right)=0
$$

for any $\epsilon>0$.

In order to discuss a similar diffusive limit, we consider a Markov chain on $\mathbb{Y}$ which is symmetric with respect to $\mathbb{M}_{\mathrm{PP}}^{(\xi)}$. Recall the down and up transition probabilities $P_{\lambda \mu}^{\downarrow(n)}$ and $P_{\mu \lambda}^{\uparrow(n-1)}$ defined in (1.3). Here the superscripts are put to make the dependence on $n$ explicit. We seek a transition probability $P_{\lambda \mu}^{(\xi)}$ which has the following form: for some $\alpha_{\xi}(n) \in(0,1)$,

$$
\begin{aligned}
& P_{\lambda \mu}^{(\xi)}=\alpha_{\xi}(n) P_{\lambda \mu}^{\uparrow(n)}+\left(1-\alpha_{\xi}(n)\right) P_{\lambda \mu}^{\downarrow(n)}, \quad \lambda \in \mathbb{Y}_{n}, n \in \mathbb{N}, \\
& P_{\emptyset \square}^{(\xi)}=\alpha_{\xi}(0), \quad P_{\emptyset \emptyset}^{(\xi)}=1-\alpha_{\xi}(0) .
\end{aligned}
$$

We ask whether it is possible to determine $\left\{\alpha_{\xi}(n)\right\}_{n=0}^{\infty}$ such that $0<\alpha_{\xi}(n)<1$ and

$$
\mathbb{M}_{\mathrm{PP}}^{(\xi)}(\lambda) P_{\lambda \mu}^{(\xi)}=\mathbb{M}_{\mathrm{PP}}^{(\xi)}(\mu) P_{\mu \lambda}^{(\xi)}, \quad \lambda, \mu \in \mathbb{Y}
$$


It suffices to verify (4.6) for $\lambda \in \mathbb{Y}_{n}$ and $\mu \in \mathbb{Y}_{n+1}$ such that $\lambda \nearrow \mu$. Combining (4.5) and (4.4), we easily see that (4.6) holds if and only if

$$
\alpha_{\xi}(n+1)=1-\frac{n+1}{\xi} \alpha_{\xi}(n), \quad n \in \mathbb{N} \sqcup\{0\} .
$$

We can observe that a solution of (4.7) is given by

$$
\alpha_{\xi}(n)=\sum_{l=0}^{\infty} \frac{(-1)^{l} \xi^{l+1}}{(n+1) \cdots(n+l+1)}, \quad n \in \mathbb{N} \sqcup\{0\}
$$

(where the series expression is essentially a special case of a Kummer function). Successive integration by parts yields

$$
\alpha_{\xi}(n)=\int_{0}^{1} \xi e^{-\xi x}(1-x)^{n} d x, \quad n \in \mathbb{N} \sqcup\{0\} .
$$

For any $\xi>0$ and $n \in \mathbb{N}$, we see from (4.9) that

$$
0<\alpha_{\xi}(n)<\alpha_{\xi}(n-1)<\alpha_{\xi}(0)=1-e^{-\xi}<1 .
$$

We have thus obtained the following.

Proposition 4.2. For $\xi>0$ define $P^{(\xi)}=\left(P_{\lambda \mu}^{(\xi)}\right)_{\lambda, \mu \in \mathbb{Y}}$ by (4.5) and (4.9) (or (4.8)). Then $P^{(\xi)}$ gives a transition matrix. A Markov chain on $\mathbb{Y}$ with transition matrix $P^{(\xi)}$ is symmetric with respect to $\mathbb{M}_{\mathrm{PP}}^{(\xi)}$, that is, it satisfies (4.6).

Remark 4.3. In the poissonized Plancherel ensemble, the typical size of a Young diagram is $\xi$, the mean of $p_{\xi}$. For $n=\xi$, (4.9) yields

$$
\alpha_{n}(n)=\int_{0}^{1} n e^{-n x}(1-x)^{n} d x=\int_{0}^{\infty} e^{-x}\left(1-\frac{x}{n}\right)^{n} 1_{[0, n]}(x) d x \stackrel{n \rightarrow \infty}{\longrightarrow} \frac{1}{2} .
$$

In other words, creation and annihilation of boxes are asymptotically balanced near the typical size.

Finally, we give an expression making the structure of the transition matrix $P^{(\xi)}$ more transparent. Set a total order in $\mathbb{Y}=\bigsqcup_{n=0}^{\infty} \mathbb{Y}_{n}$ where the number of boxes is non-decreasing and it coincides with the lexicographic order, for example, in each $\mathbb{Y}_{n}$. Set

$$
\begin{aligned}
& \Delta^{(\xi)}=\operatorname{diag}\left(\Delta_{\lambda}^{(\xi)}\right)_{\lambda \in \mathbb{Y}}, \quad \Delta_{\lambda}^{(\xi)}=\sqrt{\mathbb{M}_{\mathrm{PP}}^{(\xi)}(\lambda)}=\frac{e^{-\xi / 2} \xi^{|\lambda| / 2} \operatorname{dim} \lambda}{|\lambda| !}, \\
& A^{(\xi)}=\operatorname{diag}\left(\alpha_{\xi}(|\lambda|)\right)_{\lambda \in \mathbb{Y}} .
\end{aligned}
$$


Define $T=\left(T_{\lambda \mu}\right)_{\lambda, \mu \in \mathbb{Y}}$ by

$$
T_{\lambda \mu}= \begin{cases}1, & \lambda \nearrow \mu \\ 0, & \text { otherwise. }\end{cases}
$$

Then, $T+T^{*}$ is the adjacency matrix of the Young graph. As is well-known and easily verified, it satisfies CCR: $T T^{*}-T^{*} T=I$. Furthermore, set

$$
\left(E_{\emptyset}\right)_{\lambda \mu}= \begin{cases}1, & \lambda=\mu=\emptyset \\ 0, & \text { otherwise. }\end{cases}
$$

The following formula is directly verified.

Proposition 4.4. Under the above notation,

$$
P^{(\xi)}=\Delta^{(\xi)-1}\left\{\frac{1}{\sqrt{\xi}}\left(A^{(\xi)} T+T^{*} A^{(\xi)}\right)+e^{-\xi} E_{\emptyset}\right\} \Delta^{(\xi)} .
$$

We note that $\frac{1}{\sqrt{\xi}}\left(A^{(\xi)} T+T^{*} A^{(\xi)}\right)$ is a far-reaching analogue of a tridiagonal infinite Jacobi matrix

$$
\left(\begin{array}{ccccc}
0 & a_{1} & & & \\
a_{1} & 0 & a_{2} & & \\
& a_{2} & 0 & a_{3} & \\
& & a_{3} & \ddots & \ddots \\
& & & \ddots & \ddots
\end{array}\right),
$$

and its asymptotic spectral analysis seems to be of independent interest. We refer to [6] for such asymptotic problems about Jacobi matrices and adjacency matrices of several sorts of graphs.

Remark 4.5. In order to consider a scaling limit as in Theorem 1.4 in the grand canonical setting, we have to treat

$$
\mathbb{M}_{t}^{(\xi)}(\cdot)=\sum_{\lambda \in \mathbb{Y}} \mathbb{M}_{0}^{(\xi)}(\lambda)\left(e^{t \xi\left(P^{(\xi)}-I\right)}\right)_{\lambda}
$$

as a distribution on $\mathbb{Y}$ at macroscopic time $t>0$ and asymptotic behavior of a quantity as in (4.3) rescaled by $1 / \sqrt{\xi}$ with respect to $\mathbb{M}_{t}^{(\xi)}$ as $\xi \rightarrow \infty$. Microscopic time $t \xi$ is of the same order as the typical size $\xi$ of Young diagrams as $\xi \rightarrow \infty$. This gives rise to a difficulty because we should take into account a wide range of Young graphs which the microscopic chain driven by $P^{(\xi)}$ can spread. 


\section{Acknowledgements}

I am grateful to the editor and the referees. Thanks to their helpful comments, the manuscript was polished especially in improving the presentation and structure.

\section{References}

[1] P. Biane, Representations of symmetric groups and free probability, Adv. Math. 138 (1998), 126-181. Zbl 0927.20008 MR 1644993

[2] Approximate factorization and concentration for characters of symmetric groups, Int. Math. Res. Notices 2001, 179-192. Z Zbl 1106.20304 MR 1813797

[3] A. Borodin, A. Okounkov and G. Olshanski, Asymptotics of Plancherel measures for symmetric groups, J. Amer. Math. Soc. 13 (2000), 481-515. Zbl 0938.05061 MR 1758751

[4] A. Borodin and G. Olshanski, Infinite-dimensional diffusions as limits of random walks on partitions, Probab. Theory Related Fields 144 (2009), 281-318. Zbl 1163.60036 MR 2480792

[5] T. Funaki and M. Sasada, Hydrodynamic limit for an evolutional model of two-dimensional Young diagrams, Comm. Math. Phys. 299 (2010), 335-363. Zbl 1204.82005 MR 2679814

[6] A. Hora and N. Obata, Quantum probability and spectral analysis of graphs, Theoret. Math. Phys., Springer, 2007. Zbl 1141.81005 MR 2316893

[7] V. Ivanov and G. Olshanski, Kerov's central limit theorem for the Plancherel measure on Young diagrams, in Symmetric functions 2001, S. Fomin (ed.), Kluwer, 2002, 93-151. Zbl 1016.05073 MR 2059361

[8] S. Kerov, Interlacing measures, Amer. Math. Soc. Transl. (2) 181 (1998), 35-83. Zbl 0890.05074 MR 1618739

[9] B. F. Logan and L. A. Shepp, A variational problem for random Young tableaux, Adv. Math. 26 (1977), 206-222. Zbl 0363.62068 MR 1417317

[10] A. Nica and R. Speicher, Lectures on the combinatorics of free probability, London Math. Soc. Lecture Note Ser. 335, Cambridge Univ. Press, 2006. Z Zbl 1133.60003 MR 2266879

[11] A. Rybko, S. Shlosman and A. Vladimirov, Spontaneous resonances and the coherent states of the queuing networks, J. Statist. Phys. 134 (2009), 67-104. Zbl 1174.82005 MR 2489495

[12] P. Śniady, Gaussian fluctuations of characters of symmetric groups and of Young diagrams, Probab. Theory Related Fields 136 (2006), 263-297. Zbl 1104.46035 MR 2240789

[13] A. M. Vershik and S. V. Kerov, Asymptotic behavior of the Plancherel measure of the symmetric group and the limit form of Young tableaux, Dokl. Akad. Nauk SSSR 233 (1977), 1024-1027 (in Russian); English transl.: Soviet Math. Dokl. 233 (1977), 527-531. Zbl 0406.05008 MR 0480398 